\title{
Effect of thermal Processing Time on the Physicochemical and Sensory Properties of Lebanese Tfayfiha Verjuice Variety
}

\author{
Bayan El-kaderi ${ }^{1}$, Sami Tlais ${ }^{1}$, Ali Alkhatib ${ }^{2}$, and Rasha Hamdan ${ }^{1,3, *}$ \\ ${ }^{1}$ Lebanese International University, Nutrition and Food Sciences Department, P.O. Box: 5 Jeb-jenine Bekaa, Lebanon
${ }^{2}$ The International University of Beirut, Lebanon
${ }^{3}$ Lebanese University, Faculty of Science, Lebanon
${ }^{*}$ Corresponding author
}

Abstract - Verjuice is an unfermented, acidic, and sour-tasting juice abundant in beneficial bioactive compounds obtained from the mechanical pressing of unripe grapes. The effect of prolonged traditional thermal processing (for 0, 0.5, 1, 2, 3, 4, and 5 hours) on the physicochemical properties of 'Tfayfiha' verjuice was investigated. Polyphenol content and antioxidant capacity increased reaching their maximum $(8348.6 \mathrm{mg} / \mathrm{L})$ at 4 hours and $(77.30 \%)$ at 3 hours respectively; indicating that the ideal processing time to obtain a nutritious functional food ingredient with high bioactivity is between 3-4 hours. At 5 hours, verjuice had the lowest $\mathrm{pH}$ (1.09), lowest moisture content (26.94\%), and thus the highest shelf-life, density (1.187 $\mathrm{g} / \mathrm{mL})$, soluble solids content $\left(60.06^{\circ} \mathrm{Brix}\right)$, color intensity, and sensorial scores on the preference test. Caloric content was below detection limits (in all samples) and thus neglected. Verjuice boiled for 1 hour can be used as an excellent alternative to fresh lemon juice, where only $16.6 \%$ of the participants recognized the difference in taste between tabbouli made with fresh lemon juice and that made with verjuice boiled for 1 hour. It can be concluded that different virgins of verjuice could possibly be released to the Lebanese market by changing waste unripe grape during thinning period into a valuable product.

Keywords - verjuice, Lebanon, unripe, grapes, processing, time.

\section{INTRODUCTION}

Verjuice is an unfermented, acidic, and sour-tasting juice obtained from the mechanical pressing of the unripe grapes [1]. It is mainly consumed in the Mediterranean and southeastern regions of Turkey to enhance the flavor of traditional foods such as salads and appetizers. It is also added as an ingredient in the production of several products such as mustard sausage and various beverages [2]. It is mainly used as an alternative to lemon juice and/or vinegars. Fresh verjuice can be thermally processed into different products like sour grape sauce and unripe grape syrup or molasses [3]. It is known to contain an abundant amount of bioactive compounds of different solubility, molecular weights, structural characteristics, and intermolecular complexion [4]. Unripe grape products are widely used as acidifying and flavoring agents in the food industry and are also considered as natural antimicrobial agents on foodborne pathogens [5]. Unripe grape products have self-protection systems against some foodborne pathogens like E. coli, S. aureus, and S. typhimurium and thus could be considered as "microbiologically safe" natural products [3]. Several studies have been conducted to investigate the impact of verjuice on human health.
Verjuice is assumed to have cardio-protective properties due to its richness in bioactive compounds and its extensive phytochemical profile. It is also proposed to elicit beneficial changes to serum lipid profile, blood pressure, inflammatory markers, oxidation, glycemic control, and fatty streak formation [1]. In hyperlipidemic and hyperlipidemic with hypertensive subjects, administration of verjuice after 4 weeks resulted in significant reduction of blood pressure, LDL-C, TG, and TC concentrations [6]. Verjuice can be processed into different products depending on the duration of boiling. Sour grape sauce, for example, is heated for a short period of time ( $5 \mathrm{~min}$ ) whereas sour grape molasses can be boiled for up to 6 hours until it becomes very thick in texture [3]. According to Hayoglu's study, the chemical and sensory properties of verjuice on two varieties of unripe grapes, the natural verjuice that is neither clarified with gelatin nor heated had more flavor [2]. There is no doubt that exogenous factors affect the development of grapes $[7,8,9,10]$. A large proportion of vineyards are found in regions that are characterized by Mediterranean type climates where seasonal droughts, soil, and high 
temperature have a direct and large impact on fruit quality and yield [11].

\section{MATERIALS AND METHODS}

\subsection{Reagents}

The SIGMA Folin \& Ciocalteu's phenol reagent was used to measure the polyphenol content. The "Fluka" Hydrogen peroxide $\mathrm{H}_{2} \mathrm{O}_{2}$ solution (30\%), A.R. di-sodium hydrogen phosphate anhydrous, and Sodium phosphate monobasic anhydrous were used for the antioxidant test. The unripe grapes of the Tfayfiha variety used to conduct the physicochemical and sensory tests were harvested from a land characterized by a red soil with a $\mathrm{pH}$ of 8.3 in the West Bekaa-Lebanon on July $13^{\text {th }}, 2017$ and were completely processed and packaged on July $18^{\text {th }}, 2017$.

\subsection{Processing method}

Unripe grapes were harvested and the grape bunches were soaked in clean water for a couple of minutes, washed under tap water, then left to drain in plastic strainers. The berries were picked manually and transferred to the mill. They were grinded and mechanically pressed several times to maximize juice yield. The pomace (skins and seeds) was left aside. Fresh verjuice obtained was transferred to a large metal cooker and brought to a boil approximately at $100^{\circ} \mathrm{C}$. The foam on the top was removed progressively from time to time. In addition to the fresh sample (P0), different processed samples were prepared. The volume of the prepared samples which were boiled for different times (0.5 (P0.5), 1 (P1), 2 (P2), 3 (P3), 4 (P4), and 5 hours (P5)), was reduced by $5.4 \%, 13.5 \%, 18.9 \%, 27.0 \%, 51.4 \%$, and $78.4 \%$ respectively. Samples were packed in aseptic glass jars that were rinsed with soap and washed with clean water then boiled with their lids in water for about 15 minutes, and air-dried. The jars were firmly closed to prevent any oxygen penetration then stored at ambient temperature.

\subsection{Equipments}

The absorbance of samples was measured using a "Thermo Genesys 10-S" spectrophotometer. Samples were incubated in an "InDELAB IDL-CI-36" oven. The total soluble solids concentration was measured using a "REF107 0-90\% Brix" manual refractometer. The refractometer was calibrated with 5\% sugar solution [12]. The $\mathrm{pH}$ of the samples was measured using a "HANNA edge" HI2002-01 digital pH-meter. The moisture content was measured using a "RADWAG MA210.R" moisture content analyzer. A CAL3K-IU" oxygen bomb calorimeter was used to estimate the caloric content of the verjuice samples.

\subsection{Polyphenols measurement}

The folin test was used to measure the amount of polyphenols in verjuice extracts and gallic acid was used as standard reagent. The verjuice samples were filtered using a filter paper placed in a funnel over an Erlenmeyer flask. A blank solution is prepared by adding $20 \mu \mathrm{l}$ of $\mathrm{CO}_{2}$-free distilled water to a plastic cuvette. Add $300 \mu \mathrm{l}$ of $\mathrm{NaCO} 3$, $100 \mu 1$ of Folin-C reagent, and $1.58 \mathrm{ml} \mathrm{CO} 2$-free distilled water to each of the cuvettes. After preparing all cuvettes, they were slightly stirred and incubated in the oven at $50^{\circ} \mathrm{C}$ for 5 minutes, then removed to cool for 30 minutes. Finally, the absorbance of the prepared 10 cuvettes was measured spectrophotometrically at a wavelength of 765 $\mathrm{nm}$ [13].

\subsection{Antioxidant activity}

The hydrogen peroxide $\left(\mathrm{H}_{2} \mathrm{O}_{2}\right)$ scavenging assay can be used. The ability of plant extracts to scavenge hydrogen peroxide can be estimated by using the Ruch method [14]. A solution of hydrogen peroxide $(40 \mathrm{mM})$ was prepared in phosphate buffer (50 $\mathrm{mM}$ and $\mathrm{pH}=7.4)$. The concentration of hydrogen peroxide was determined by the absorption at $230 \mathrm{~nm}$ using a spectrophotometer. Extract $(20-60 \mu \mathrm{g} /$ $\mathrm{mL}$ ) in distilled water was added to $\mathrm{H}_{2} \mathrm{O}_{2}$ and absorbance at $230 \mathrm{~nm}$ was determined after $10 \mathrm{~min}$ against a blank solution containing phosphate buffer without hydrogen peroxide.

\subsection{Sensory evaluation}

A traditional Lebanese food called Tabbouli salad was prepared by mixing parsley (532 g), tomatoes (768 g), cucumber $(350 \mathrm{~g})$, burghul (1.5 cups), olive oil $(240 \mathrm{ml})$, salt (3 Tbsp), and dried mint (1.5 Tbsp). Equal amount (75 $\mathrm{ml}$ ) of lemon juice and P1 verjuice were added to the Tabbouli plates.

\subsubsection{Triangle test}

The performed test was a triangle test that seeks whether the participants can differentiate between sour grape sauce and lemon juice in a traditional Lebanese food called tabbouli, thus its possible use as an alternative to fresh lemon juice. Preference test was carried out by 12 trained Lebanese participants ( 8 females and 4 males) aged 18 and above. Each person was given 3 coded samples of tabbouli and was informed that two of the three samples are identical and one is odd. The samples presented were either 2 made with lemon juice and 1 with verjuice or 2 made with verjuice and 1 with lemon juice. They were asked to drink water and/or eat bread between each trial. The 6 possible combinations (AAB, BAA, ABA, BBA, BAB, $\mathrm{ABB})$ were prepared and randomly presented to subjects (twice). 


\subsubsection{Preference test}

A total of 50 trained Lebanese subjects (30 females and 20 males) aged 18 years and above participated in the test. The subjects were given 3 coded samples in small plastic cups each boiled for a different duration of time (sample $1=30 \mathrm{~min}$, sample $2=3 \mathrm{hrs}$, \& sample $3=5 \mathrm{hrs}$ ). They were asked to rate the sourness, color, overall flavor acceptability, and the samples' overall acceptability as an alternative to lemon juice. Participants were given bread to eat and water to sip on between each sample.

\subsection{Statistical Analysis}

The means of different results of verjuice samples were separated by the SPSS (Statistical Package for the Social Sciences, version 22.0) program. Analysis of variance consisted of univariate analysis through Tukey's Honest Significant Differences test $(\alpha=0.05)$. Tukey's Honest Significant Difference test was used to compare means between processed verjuice samples in physiochemical tests and the preference test. In addition, the Pearson Correlation Test was conducted to seek the relationship between the different parameters tested. In Tukey's Honest Significant Differences test ( $\alpha=0.05$ ), letters a, b, c, and d, denoted on different result means, indicate these mean values are significantly different $(\mathrm{p}=5 \%)$ when they are denoted with different letters, and non-significantly different when they are denoted with a common letter. Mean values are denoted with letters in a descending order where "a" is denoted to the highest mean value and " $\mathrm{g}$ " to the lowest mean value.

\section{RESULTS AND DISCUSSION}

\subsection{Polyphenol content}

Verjuice is known to contain an abundant amount of bioactive compounds such as flavonoids, phenolic acids, hydroxycinnamic acids, all of which contribute to extensive health benefits [1]. In our study, the polyphenol contents of the 7 verjuice samples steadily increased with the progression of heat treatment and ranged between 1189.2 and $8348.6 \mathrm{mg} / \mathrm{L}$. The polyphenol contents of the 7 verjuice samples (P0, P0.5, P1, P2, P3, P4, and P5) were determined by the Folin Ciocalteu assay (Fig. 1). Although the samples showed an obvious increase in polyphenol content with processing time, the processed samples P0.5, $\mathrm{P} 1$, and P2 were not significantly different from the fresh (raw) verjuice sample. In general, polyphenol content ranged between $1189.2 \pm 209.0 \mathrm{mg} / \mathrm{L}$ and $8348.6 \pm 525.5$ $\mathrm{mg} / \mathrm{L}$.

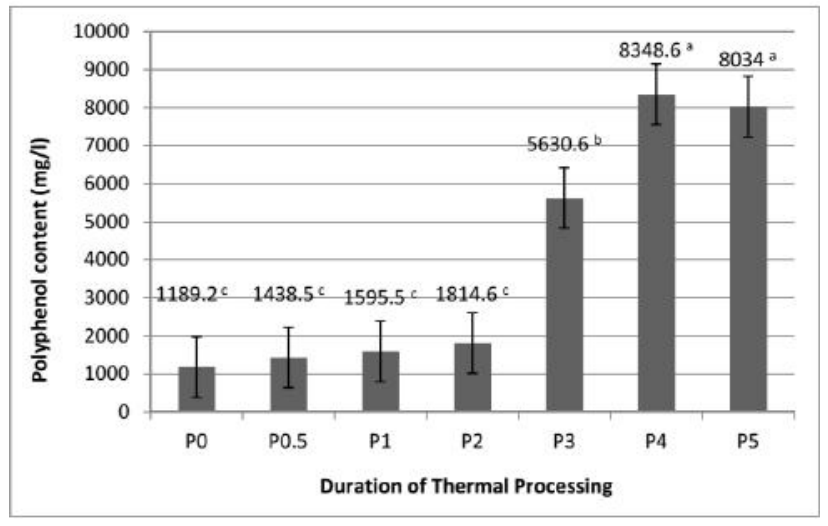

*Means with different letters are significantly different at $\mathrm{p}=0.05$ according to Tukey's test.

Fig. 1: Polyphenol contents in $\mathrm{mg} / \mathrm{L}$ of the fresh and processed verjuice samples

The concentration of polyphenols continued to increase with processing time with a sharp increase at 3 and 4 hours (samples P3 and P4 respectively) and then came to a decrease at 5 hours (sample P5) where the polyphenol content dropped from $8348.0 \pm 525.5 \mathrm{mg} / \mathrm{L}$ to 8034.0 $\pm 794.0 \mathrm{mg} / \mathrm{L}$. However, samples P4 and P5 were not considered significantly different but polyphenols decreased by $314.6 \mathrm{mg}$. In Oncul's study on verjuice, the total polyphenol concentration ranged between 233.44 and $672.75 \mathrm{mg} / \mathrm{L}$ which is lower than our results [3]. Similarly, American Rootstock verjuice had a total phenol content of $652.13 \mathrm{mg} / \mathrm{L}$ [3]. Moreover, Lee and Talcot (2004) determined the phenolic content of unripe grape samples to be between 739 and $1673 \mathrm{mg} / \mathrm{L}$. Hayoglu, however, found that verjuice obtained from Kabarcik unripe grapes had a lower phenol content $(6262.7 \mathrm{mg} / \mathrm{L})$ than that obtained from Yediveren variety $(7538 \mathrm{mg} / \mathrm{L})$ [2]. This wide variation in polyphenol content can be related to many factors like geographical origin, maturity, agricultural practices, genotype of grape, variety, time of harvest, amount of grape used, environmental factors, nutrition and water status $[15,16]$. In fact, general conclusions could not be drawn because it was obvious that thermal treatment can have a differential effect on polyphenol levels [17]; where in our study, thermal treatment resulted in an increase of polyphenols to a certain point (maximum at 4 hours) and then a slight decrease afterwards.

\subsection{Total soluble solids (TSS)}

In general, at the unripe stage of grape development, the main soluble solids are phenolic compounds, glucose, fructose, malic, tartaric, and citric acid [18]. The proportion of each compound can be influenced by genotype and environmental factors. In our study, fresh verjuice had a soluble solids value of $5.93^{\circ}$ Brix and was shown to increase with the progression of thermal 
treatment (fig. 2). Up to 1 hour of boiling, samples P0.5 and P1 showed a slight but nonsignificant increase in the concentration of soluble solids (6.33 and $6.73^{\circ}$ Brix respectively). In Oncul's study, the total soluble solids (TSS) content varied significantly between 3.55 and $8.00^{\circ}$ Brix [3]. Moreover, Hayoglu reported a significant difference in the TSS content between the two varieties Kabarcik $\left(7.47^{\circ}\right.$ Brix) and Yediveren $\left(4.50^{\circ}\right.$ Brix). This indicates that the differences in TSS content can be largely attributed to the differences in variety and genotype [2]. In addition, these differences may also be associated with the time of harvest and the sensitivity of each variety to the growing conditions and delays in crop sampling [18]. Thus, an ideal harvesting time should be determined to obtain a higher quality of verjuice and a more consistent final product in the market. Moreover, it has been shown that soils containing less water and organic matter yielded higher total soluble solids and contributed to the excellent quality of grapes [10]. A significant increase in TSS content was observed in our samples at 2 hours of boiling and above reaching a maximum concentration at 5 hours $\left(60.06^{\circ}\right.$ Brix). The pattern of TSS content increase can be aligned with the moisture and polyphenol content results where significant changes were also observed after 2 hours of boiling.This can be due to a smaller reduction of bioactive compounds and thus an increased concentration of nonvolatile thermo-stable organic compounds (Saenz, 2010)

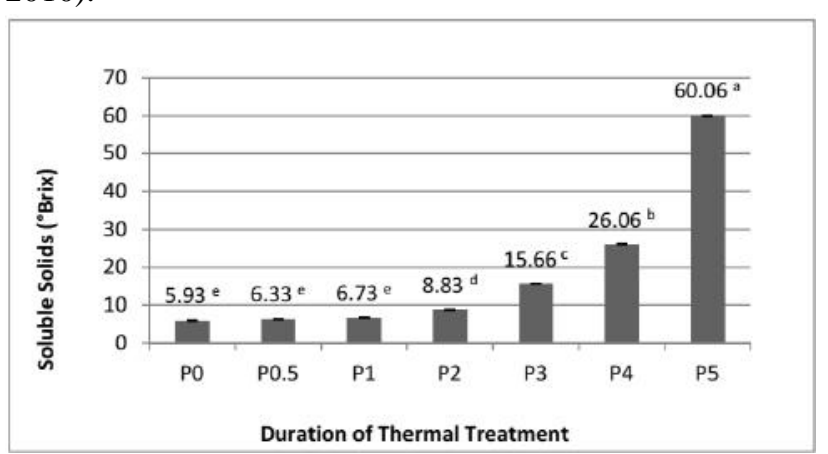

*Means with different letters are significantly different at $(\mathrm{p}=0.05)$ according to Tukey's test

Fig. 2: Soluble solids values in ${ }^{\circ}$ Brix with prolonged thermal processing

\subsection{Density}

The fresh verjuice sample had a density of $0.81 \mathrm{~g} / \mathrm{mL}$. As the duration of thermal processing increased up to 3 hours, the density began to slightly increase (Fig. 3). The values were not significantly different until reaching 4 and 5 hours of boiling ( 0.90 and $1.18 \mathrm{~g} / \mathrm{mL}$ respectively) where the samples had a thick texture. Density measurements showed conformity with the moisture content results where the significant change was observed after 2 hours of thermal processing $(\mathrm{r}=-0.966, \mathrm{p}<0.01)$. Zuritz found that density was correlated to the concentration of total soluble solids and temperature [19]. In fact, with the progression of thermal processing, there was an increased production of the polyphenols and thus a higher value of TSS. In addition, insoluble solids of verjuice can also contribute to the density values.

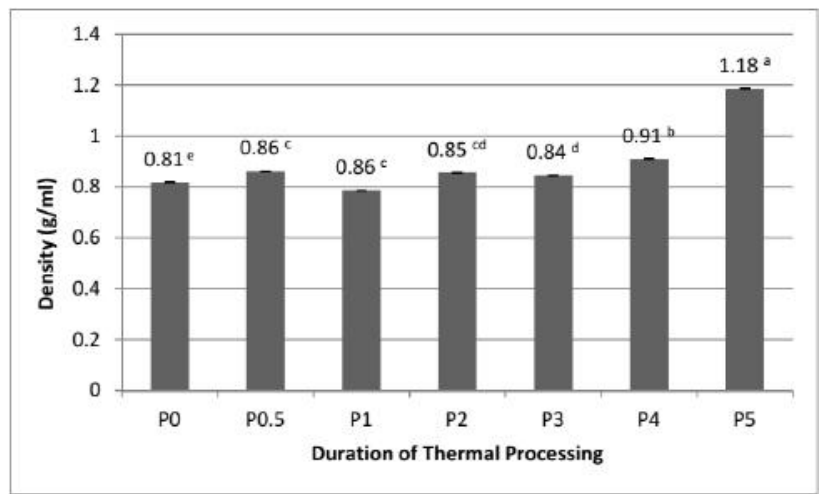

*Means with different letters are significantly different at $(\mathrm{p}=0.05)$ according to Tukey's test

Fig. 3: The density values of the fresh and processed verjuice samples at different processing times in $\mathrm{g} / \mathrm{mL}$

\subsection{Color intensity}

Color is one of the most important sensory attributes and the first characteristic a consumer notices when using or buying a food product. Thermal processing at high temperatures is usually used in the industry to provide food different taste, aroma, and color; all of which are desirable changes that enhance the quality of foodstuffs $\left[{ }^{20}\right]$. In this study, it was shown that color intensity increased with longer thermal processing. The absorbance steadily increased and ranged between 0.078 and 1.145 for the first 4 samples (P0 to P2) (Fig. 4). However, samples P4 and P5 were very concentrated and could not be detected spectrophotometrically. Visually, the fresh sample was very turbid and had a dark greenish color. After 30 minutes of boiling, the solution became clearer and changed to a yellowish color. The color began to change from a bright orange-to-brown after 1 hour of boiling to a darker brownto-red shade after 2 hours. Afterwards, the verjuice samples turned very dark in color as they became more concentrated. This can be explained by the last stage of the Maillard reaction when aldol condensation occurs and highly colored heterocyclic nitrogenous compounds are formed such as melanoidins [21]. In addition, caramelization can also be a reason for change in color where simple sugars (in the absence of amino acids) are degraded with heat processing and result in a brownish shade [22]. Irena reported a positive linear correlation between gallic, vanillic, ferulic, and protocatechuic acid 
content and color intensity in posip Rukatac wines [16]. Moreover, Ashoush found that browning was more evident in the atmospheric heating method while preparing pomegranate juice concentrate compared to microwave heating [23]. Knowing that traditional (atmospheric) thermal processing was exploited in our experiment, a higher browning effect can be associated with enzymatic browning at least in the early stages of the process before the complete inactivation of enzymes. In this method, there is a prolonged exposure to oxygen throughout the whole process, thus higher oxidation of phenolic compounds by polyphenoloxidases (PPO) that results in a change in the color parameters of the samples.

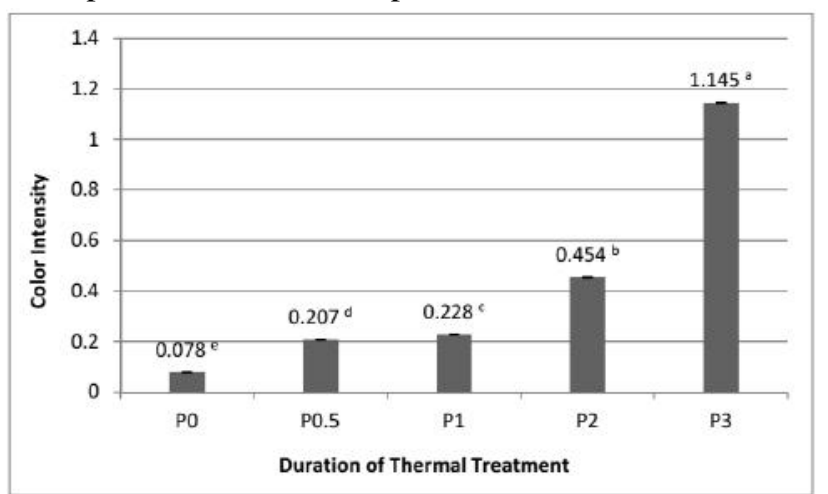

*Means with different letters are significantly different at $(\mathrm{p}=0.05)$ according to Tukey's test

Fig. 4: The color intensity (absorbance) values at $\lambda=520$ $n m$ of the first five samples of verjuice

\section{6. $\mathrm{pH}$ Measurements}

The $\mathrm{pH}$ values were observed to steadily decrease with the application of traditional thermal processing (fig. 5). After 30 minutes of boiling, the $\mathrm{pH}$ value of the fresh verjuice sample significantly decreased from 2.60 to 2.45 and continued to decrease with an increase in processing time to reach a minimum value of 1.09 (after 5 hours). The $\mathrm{pH}$ of verjuice before thermal processing was 2.60 being the highest among all the samples. In Oncul's study on five verjuice and five sour grape sauce samples of different varieties, the $\mathrm{pH}$ of verjuice samples ranged between 2.35 and 2.59 which is almost similar to our results [3]. However, Hayoglu found that $\mathrm{pH}$ of verjuice obtained from Kabarcik and Yediveren varieties were high (2.98 and 2.91 respectively) [2]. This variation between different verjuice samples can obviously be related to the genotypic and variety differences of the unripe grapes investigated. Moreover, the growing conditions, soil types, time of harvest, maturation stage, and environmental stresses can also contribute to the differences in $\mathrm{pH}$ values [3].

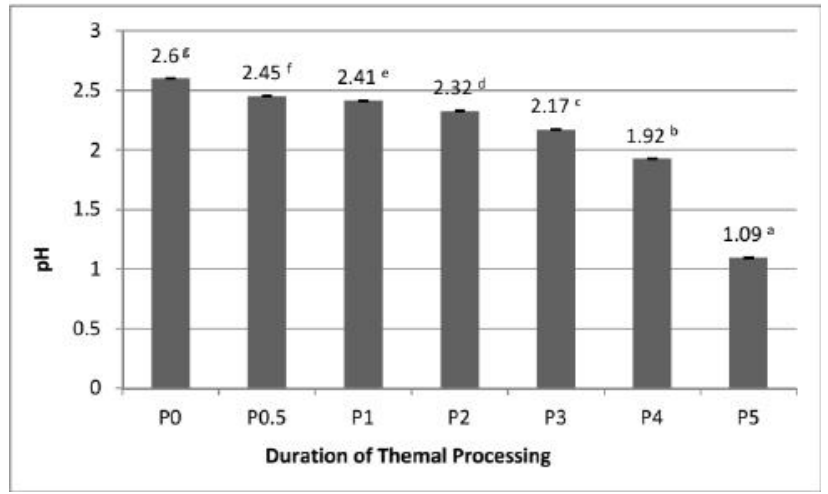

*Means with different letters are significantly different at $(\mathrm{p}=0.05)$ according to Tukey's test

Fig. 5: The $\mathrm{pH}$ values of the fresh and processed verjuice samples at different thermal processing times.

\subsection{Moisture Content}

The main reason for decreasing the moisture content of a product is to lower its risk for undesirable spoilage and thus increasing its shelf-life. In addition, producing a highly concentrated version of verjuice can be more economic and of a higher sensorial quality. Fresh verjuice had a moisture content of $95.08 \%$ which makes it vulnerable to undesirable microbial spoilage (fig. 6). As the duration of thermal processing increased the moisture content began to slightly decrease until the first 2 hours of boiling. Afterwards, the moisture content began to decrease significantly until reaching a minimum value of $26.94 \%$ at 5 hours $\left(60^{\circ}\right.$ Brix $)$. The main process taking place during moisture decrease is evaporation.

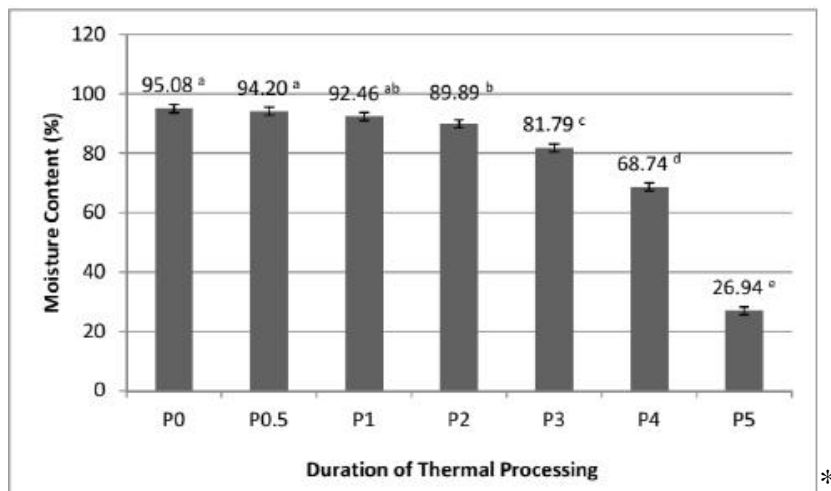

Means with different letters are significantly different ( $\mathrm{p}=5 \%$ ) according to Tukey's test

Fig. 6: Moisture content values of the verjuice samples

\subsection{Antioxidant capacity}

The antioxidant activity was measured using the $\mathrm{H}_{2} \mathrm{O}_{2}$ scavenging assay (Fig. 7). The fresh verjuice sample had a $67.91 \%$ scavenging activity; and as the duration of thermal processing increased, the scavenging activities of the samples were shown to significantly increase as well (up to 
3 hours of boiling). At hour 4 , the scavenging activity began to decline $(70.05 \%)$ reaching a minimum of $53.61 \%$ at 5 hours. It has been previously reported that there is a high correlation between phenolic compound content and antioxidant capacity in fruits and vegetables [24]. In our study the polyphenol content and TSS of the fresh verjuice sample increased with the progression of processing. These findings indicate that total soluble solids including polyphenols are the major contributors to antioxidant activity $(r=-0.786, p<0.05)$, and the factors contributing to their loss in the samples are the same as those resulting in antioxidant capacity diminution [25]. In grape seed, the total phenol content and subsequent antioxidant capacity significantly increased with heat treatment $\left(150^{\circ} \mathrm{C}\right.$ for 40 minutes) compared to unheated samples [26]. Moreover, Pinelo found that the total and antioxidant capacity of grape extract increased with heat treatment and was associated with the formation of oligomers from free polyphenols [27]. In another study, the heat treatment of grape seeds significantly increased the contents of gallocatechin, gallate, and caffeine in their extracts and thus were the major contributors to the increase in antioxidant capacity. It was assumed that insoluble phenolic compounds were extracted upon heat treatment [24. It was reported by Helvacioglu that samples produced by traditional methods had the highest antioxidant capacity [28]. Moreover, the total antioxidant capacity and $\mathrm{H}_{2} \mathrm{O}_{2}$ scavenging activity of grape molasses produced by traditional techniques were higher compared to samples produced by modern techniques practiced in the industry. Depending on the degree of thermal treatment applied, antioxidants can be degraded and new components with antioxidant capacity can be formed [29]. However, the change in antioxidant capacity in samples produced by traditional methods may be explained by the magnitude and duration of the temperature used which can justify the decrease of $\mathrm{H}_{2} \mathrm{O}_{2}$ scavenging after long processing times (4 and 5 hours). Eventually, it should be noted that many other factors can influence total phenolic content and thus the antioxidant capacity in grape products such as the growing conditions, environmental factors, genotype, and post-harvest treatments [28].

\subsection{Energy content}

Verjuice samples did not record any results concerning their caloric content. It is known that unripe grapes contain a very small amount of sugar (i.e. 13.3 - $30.7 \mathrm{~g} / \mathrm{L}$ ) and the accumulation of glucose and fructose is very slow up to veraison [18]. This indicates that harvest time is a major factor that affects the sugar content of verjuice products and thus its caloric content since it does not contain any lipids and only a small amount of proteins.

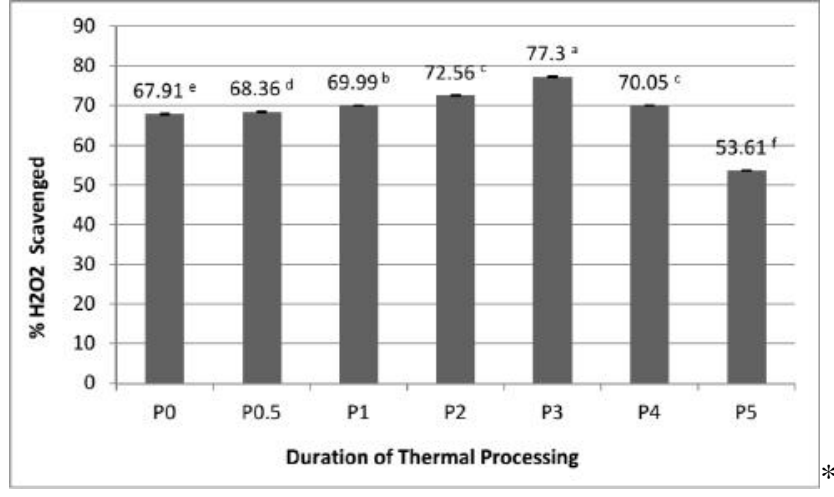

Means with different letters are significantly different ( $\mathrm{p}=5 \%$ ) according to Tukey's test

Fig.7: The percentage of hydrogen peroxide $\left(\% \mathrm{H}_{2} \mathrm{O}_{2}\right)$ scavenged at different thermal processing times.

\subsection{Sensory evaluation}

\subsubsection{Preference test}

For P0.5, the sourness had an average of 2.68 (between fair and good), the color 2.98, the overall flavor 3.38, and an overall acceptability as an alternative to lemon juice of 3.06 (Table 1).

Table 1. Preference test scores of the three verjuice samples tested

\begin{tabular}{|c|c|c|c|c|}
\hline $\begin{array}{l}\text { Sample } \\
\text { ID }\end{array}$ & Sourness & Color & flavor & $\begin{array}{l}\text { Alternative } \\
\text { to lemon } \\
\text { juice }\end{array}$ \\
\hline P0.5 & $2.68^{a} \pm 0.93$ & $2.98^{a} \pm 1.09$ & $3.38^{\mathrm{a}} \pm 0.80$ & $3.06^{b} \pm 1.16$ \\
\hline P3 & $4.02^{b} \pm 0.37$ & $3.18^{a} \pm 0.77$ & $3.36^{a} \pm 0.69$ & $3.70^{a} \pm 0.88$ \\
\hline P5 & $4.92^{c} \pm 0.27$ & $4.32^{b} \pm 0.97$ & $3.78^{a} \pm 1.31$ & $3.92^{\mathrm{a}} \pm 1.39$ \\
\hline
\end{tabular}

*Means with different letters are significantly different at $(\mathrm{p}=0.05)$ according to Tukey's test

For P3, the sourness had an average of 4.02, the color 3.18, an overall flavor of 3.36, and an overall acceptability as an alternative to lemon juice of 3.70. For P5, the sourness had an average of 4.92 , a color average of 4.32 , an overall flavor score of 3.78 , and an overall acceptability as an alternative to lemon juice of 3.92. Thus, different virgins of verjuice could possibly be released as standardized products to the Lebanese market to satisfy different consumer needs and preferences.

\subsubsection{Triangle difference test}

Verjuice boiled for 1 hour was substituted with fresh lemon juice in tabbouli and only $16.6 \%$ were able to detect a difference between the samples. However, the majority $(83.3 \%)$ of panelists did not seek a perceptible difference between samples made with verjuice and those made with fresh lemon juice. Both lemon juice and verjuice (boiled 
for 1 hour) have an outstanding acidic sour taste and low $\mathrm{pH}$ (around 2). Thus, these common characteristics also make it a good alternative to lemon juice.

\section{CONCLUSION}

Traditional thermal processing had a positive effect on both the physicochemical and sensory properties of Lebanese 'Tfayfiha' verjuice. Polyphenol content and antioxidant capacity increased with time reaching their maximum $(8348.6 \mathrm{mg} / \mathrm{L})$ at 4 hours and $(77.30 \%)$ at 3 hours respectively; making processed verjuice a healthy low-calorie functional food ingredient with a relatively high bioavailability. Verjuice boiled for 1 hour can be considered as a good alternative to lemon juice in some cases i.e. in Tabbouli; possibly due to its outstanding acidic sour taste and low pH (around 2). Different virgins of verjuice could possibly be released to the Market to satisfy different consumer needs and preferences. However, several factors should be considered when producing a standard and uniform commercial verjuice product such as harvest time of unripe grapes, cultural area, and duration of thermal treatment.

\section{REFERENCES}

[1] Ahmadi L, Roney SK.(2014). Pharmacological and phytochemical properties of unripe grape juice (verjuice): A review. Austin J Nutr Metab. 1(2);9.

[2] Hayoglu I, Kola O, Kaya C, Turkoglu H.(2009). Chemical and sensory properties of verjuice a traditional turkish nonfermented beverage from Kabarcik and Yediveran grapes. Journal of Food Processing and Preservation. 33 (s1); 252263.

[3] Oncul N, Karabyikli S.(2015). Factors affecting the quality attributes of unripe grape functional food products. Journal of Food Biochemistry. 39; 689-695.

[4] Setorki M, Asgary S, Haeri Rohani A.(2010). Effects of acute verjuice consumption with a high-cholesterol diet on some biochemical risk factors of atherosclerosis in rabbits. Med Sci Monit. 16: 124-130.

[5] Seniz K, Nilgun O.(2016). Inhibitory effect of unripe grape products on foodborne pathogens. Journal of Food Processing and Preservation. 40:1459-1465.

[6] Alipour, M., Davoudi, P., Davoudi, Z.(2012).Effects of unripe grape juice (verjuice) on plasma lipid profile, blood pressure, malondialdehyde and total antioxidant capacity in normal, hyperlipidemic and hyperlipidemic with hypertensive human volunteers. Journal of Medicinal Plants Research. 6, 5677-5683.

[7] Li JM, Jiang WG, Yu Y, Liang DM, Liang HZ.(2013). Effects of different soil texture on the quality of winemaking grape and grape wine. Liquor-Making Science and Technology. 7:37-41.
[8] Wang XQ, Chen XB, Zhan JC, Huang WD.(2006). Effects of ecological factors on quality of wine grape and wine. Food Science. 27:791-797.

[9] Conradie WJ, Carey VA, Bonnardot V, Saayman D, Van Schoor LH.(2002). Effect of different environmental factors on the performance of Sauvignon blanc grapevines in the Stellenbosch/Durbanville districts of South Africa. I. Geology, soil, climate, phenology and grape composition. South African journal for enology and viticulture. 23: 78-91.

[10] Cheng G, He YN, Yue TX, Wang J, Zhang ZW.(2014). Effects of climatic conditions and soil properties on Cabernet Sauvignon berry growth and anthocyanin profiles. Molecules. 19(9): 13683-703.

[11] Chaves MM, Zarrouk O, Francisco R, Costa JM, Santos T, Regalado AP, Rodrigues ML, Lopes CM.(2010). Grapevine under deficit irrigation: hints from physiological and molecular data. Ann Bot. 105(5): 661-76.

[12] Neilson, S. S. (2010). Standard solutions and titratable acidity. Food Analysis Laboratory Manual ( $2^{\text {nd }}$ ed.). USA: Springer, pp 20-100.

[13] Singleton, V. L., Orthofer, R., \& Lamuela-Raventos R. M. (1999). Analysis of total phenols and other oxidation substrates and antioxidants by means of Folin-Ciocalteu Reagent. Methods Enzymol., 299, 152-178.

[14] Ruch R J, Cheng S J, Klaunig J E.(1989). Prevention of cytotoxicity and inhibition of intracellular communication by antioxidant catechins isolated from Chinese green tea.Carcinogenesis. 10; 1003-1008.

[15] Jeusti Bof CM, Fontana RC, Piemolini-Barreto LT, Sandri IG.(2012).Effect of freezing and processing technologies on the antioxidant capacity of fruit pulp and jelly.Braz.arch.biol.technol. 1678-4324.

[16] Irena I, Mohamed G. (2012). Biological activities and effects of food processing on flavonoids and phenolic antioxidants. Marian P, editor. Advances in applied biotechnology. InTech; p. 101-124.

[17] Szwajgier D, Halinowsk T, Helman E, Tylus K, Tymcio A.(2014). Influence of different heat treatments on the content of phenolic acids and their derivatives in selected fruits. Hum. Nutr.Sci. Food Commod. 69(2): 167-178.

[18] Sabir A, Kafkas E, Tangolar S.(2010). Distribution of major sugars, acids, and total phenols in juice of five grapevine (Vitis spp.) cultivars at different stages of berry development.Spanish Journal of Agricultural Research. 8(2): 425-433.

[19] Zuritz CA, Munoz Puntes E, Mathey HH, Perez EH, Gascon A, Rubio LA, Carullo CA, Chernikoff RE, Cabeza MS.(2005). Density, viscosity, and coefficient of thermal expansion of clear grape juice at different soluble solid concentrations and temperatures. Journal of Food Engineering. 71(2): 143-149.

[20] Tamanna N, Mahmood N.(2015). Food processing and maillard reaction products: effect on human health and nutrition. International Journal of Food Science.1-6. 
[21] Hodge JE.(1953). Dehydrated foods: chemistry of browning reactions in model systems.Journal of Agricultural and Food Chemistry. 1(15):928-943.

[22] Quintas M, Brandao TRS, Silva CLM.(2007). Modeling autocatalytic behavior of a food model system-sucrose thermal degradation at high concentrations. J Food Eng. 78:537-545

[23] Ashoush IS, Gadallah MGE.(2012). Effects of different heating methods on the quality characteristics of pomegranate juice concentrates. Egypt. J. Food Sci. 40: 114.

[24] Kim SY, Jeong SM, Park WP, Nam KC, Ahn DU, Lee SC.(2006). Effect of heating conditions of grape seeds on the antioxidant activity of grape seed extracts.Food Chem. 97:472-479.

[25] Preti R, Rapa M, Vinci G.(2017). Effect of steaming and boiling on the antioxidant properties and biogenic amines content in grean bean (phaseolus vulgaris) varieties of different colours. Journal of Food Quality. 2017(2017): 8.

[26] Ross CF, Hoye C, Fernandez-plotka VC.(2011). Influence of heating on the phenolic content and antioxidant activity of grape seed flour. J Foof Sci. 76:884-890.

[27] Pinelo M, Rubilar M, Sinerio J, Nunez MJ.(2005). A thermal treatment to increase the antioxidant capacity of natural phenols: Catechin, resveratrol, and grape extract cases. Eur Food Res Technol. 221:284-290.

[28] Helvacioglu S, Charehsaz M, Guzelmeric E, Turkoz Acar E, Yesilada E, Aydin A.(2018). Comparatively investigation of grape molasses produced by conventional and industrial techniques. Marmara Pharm J. 22(1): 44-51.

[29] Calligaris S, Manzocco L, Anese M, Nicoli MC.(2004). Effect of heat treatment on the antioxidant and pro-oxidant activity of milk. Int Dairy J. 14:421-427. 